\title{
ADAM17-siRNA inhibits MCF-7 breast cancer through EGFR-PI3K-AKT activation
}

\author{
XIANGCHAO MENG ${ }^{1}$, BAOSHAN HU $^{1}$, MOHAMMAD MONIR HOSSAIN $^{2}$, \\ GUOFU CHEN $^{1}$, YING SUN ${ }^{3}$ and XUEPENG ZHANG ${ }^{1}$
}

\author{
${ }^{1}$ Department of Surgical Oncology, Affiliated Hospital, ${ }^{2}$ International Education College, ${ }^{3}$ Department of Pathology, \\ Basic Scientific Medical College, North China University of Science and Technology, Tangshan, Hebei 063000, P.R. China
}

Received February 22, 2016; Accepted May 16, 2016

DOI: 10.3892/ijo.2016.3536

\begin{abstract}
A disintegrin and metalloproteinase-17 (ADAM17) can cut and release a wide variety of epidermal growth factor receptor (EGFR) ligands to promote survival, invasion and proliferation of cancer cell, and therefore, is considered to be a potential therapeutic target for cancer. The main goal of the present study was to observe the effects of ADAM17 small interfering RNA (ADAM17-siRNA) on human MCF-7 breast cancer and investigate its activation pathway. In vitro, MCF-7 cells were divided into ADAM17-siRNA groups, nonsense siRNA groups, AG1478 (selective EGFR blocker) groups, LY294002 [phosphatidylinositol 3-kinase (PI3K) phosphorylation inhibitor] groups, PD0325901 [mitogen extracellular kinase (MEK) inhibitor] groups and control groups. In vivo, MCF-7 cells were implanted subcutaneously into nude mice and then these mice were randomly divided into ADAM17-siRNA groups, vector groups and control groups. Our data showed that compared with the control groups, ADAM17-siRNA, AG1478 and LY294002 could inhibit the migration and proliferation of MCF-7 cells, but PD0325901 and nonsense siRNA did not show this effect. Except that specific ADAM17-siRNA could inhibit the expression of ADAM17 mRNA, others did not change it. Western blot analysis further confirmed that EGFR-PI3K-AKT signaling pathway is involved in ADAM17-siRNA inhibiting migration and proliferation of MCF-7 cells. Similarly to the former, the growth of MCF-7 breast cancer in nude mice was significantly inhibited by ADAM17-siRNA. Compared with the control group and the vector group, the tumor volume was smaller in the ADAM17-siRNA group, the tissues developed
\end{abstract}

Correspondence to: Professor Xuepeng Zhang, Department of Surgical Oncology, Affiliated Hospital, North China University of Science and Technology, No. 73 South Jianshe Road, Tangshan, Hebei 063000, P.R. China

E-mail: syzxp@sina.com

Keywords: breast cancer, small interference RNA, a disintegrin and metalloprotease 17, targeted therapy, EGFR-PI3K-AKT signaling pathway large areas of necrosis, immunohistochemistry showed low expressions of ADAM17 and Ki-67 and western blot analysis proved that the expression of ADAM17 protein in the tissue was also reduced. The present study suggests that ADAM17siRNA inhibits MCF-7 breast cancer and is activated through the EGFR-PI3K-AKT signaling pathway.

\section{Introduction}

Breast cancer is one of the most common malignant tumors in women, and its incidence rate ranks first in gynecological tumors (1). For the past few years, the main focus for research on the treatment of cancer has gradually transferred from the use of cytotoxic drugs to targeted therapies, that is, targeting specific genes or proteins that play a key role in the growth and progression of cancer (2). In this area, compared with a wide range of studies on genes or proteins that are downstream of the epidermal growth factor receptor (EGFR), corresponding work focusing on upstream genes or proteins has rarely been carried out (3). A disintegrin and metalloproteinase (ADAM) family members are a series of Zn-dependent metalloproteinases and they are, as ectodomain sheddases, best known for their domains that function as metalloproteases (4). ADAM exists in a variety of organisms and is mainly distributed in the cell membrane (5). Studies have indicated that it is closely related to tumor invasion and metastasis and plays a crucial role in the progression of breast cancer $(4,5)$. Because the 17th member of the family is the major enzyme which takes charge of releasing soluble tumor necrosis factor-alpha (TNF- $\alpha$ ) from the plasmalemma, it is also known as TNF- $\alpha$ converting enzyme (TACE/ADAM17) (6). Besides releasing TNF- $\alpha$, ADAM17 is also conducive to the progression of the disease by means of processing a number of growth factors and growth factor receptors and takes part in the activation of EGFR and related receptors, which is causally related to the progression of different cancers of epidermal origin (6-8). ADAM17 plays the role of signal scissor in cancer microenvironment (9). Recently, it has been proved as the major sheddase for a variety of EGFR pro-ligands such as heparinbinding-EGF, transforming growth factor-alpha (TGF- $\alpha$ ), amphiregulin (AREG), neuregulin (NRG), epiregulin (EREG) and betacellulin (BTC) as well as factors which are important in inflammation, particularly TNF- $\alpha$ and its receptor $(2,10,11)$. 
EGFR ligand-binding leads to receptor self-dimerization, autophosphorylation and followed activation of downstream MEK-ERK or PI3K-AKT pathways $(12,13)$. In the field of breast cancer, there has been substantial research on the expression of ADAM17 based on cells, animal models and clinical samples. ADAM17 showed a lower expression in less aggressive rather than in highly aggressive breast cancers and patients with low ADAM17 expression had an obviously longer overall survival than those with high expression $(14,15)$. Our previous studies found that there was a significant increase of ADAM17 expression in breast cancers compared to normal human breast tissues $(16,17)$, which indicate that ADAM17 may be a potential clinical therapeutic target for breast cancer, and that ADAM17-siRNA inhibited MCF-7 breast cancer cell proliferation and invasion in vitro $(18,19)$. Our present study reports that ADAM17-siRNA inhibits MCF-7 breast cancer cell migration and proliferation in vitro and is activated through the EGFR-PI3K-AKT signaling pathway and that ADAM17-siRNA can inhibit MCF-7 breast cancer in vivo.

\section{Materials and methods}

Cell line and cell culture. The MCF-7 human breast cancer cell line was obtained from the Institute of Basic Medical Sciences, Chinese Academy of Medical Sciences (Beijing, China). The cells were cultured in Dulbecco's modified Eagle's medium (DMEM; Invitrogen, Carlsbad, CA, USA) supplemented with $10 \%$ fetal bovine serum (FBS), $50 \mu \mathrm{g} / \mathrm{ml}$ streptomycin and 50 units $/ \mathrm{ml}$ penicillin, in a humidified atmosphere incubator of $5 \% \mathrm{CO}_{2}$ at $37^{\circ} \mathrm{C}$. The selective EGFR blocker AG1478, PI3K phosphorylation inhibitor LY294002, and the MEK inhibitor PD0325901 were purchased from Sigma-Aldrich. Cells from AG1478, LY294002 and PD0325901 group were cultured with $20 \mu \mathrm{M}$ AG1478, LY294002 and PD0325901 for $48 \mathrm{~h}$ before migration and proliferation assay, real-time PCR and western blot analysis, respectively.

Transfection of MCF-7 cells with ADAM17-siRNA. ADAM17small interference RNA (ADAM17-siRNA): 5'-TGAGGCAG TCTCTCCTATTCCTGACCAGC-3' and nonsense siRNA: 5'-TGACCACCCTGACCTACGGCGTGCAGTGC-3' were from Shanghai GenePharma, Co., Ltd. (Shanghai, China). MCF-7 cells of the ADAM17-siRNA groups and the nonsense siRNA groups were transfected with Lipofectamine 2000 (Invitrogen) following the manufacturer's instruction. The same amount of PBS was added to the control group cells.

Quantitative real-time polymerase chain reaction ( $q R T-P C R)$. According to the manufacturer's instruction, cells were rinsed with PBS and RNA was extracted using TRIzol reagent (Invitrogen). Subsequently, RNA was converted to cDNA with SuperScript II reverse transcriptase (Invitrogen). Whereafter, qRT-PCR was performed. Primers designed by Primer Premier 5 software were as follows, ADAM17: 5'-ATCAAA CCTTTCCTGCG-3' (forward) and 5'-CAAACCCATCCTC GTCCA-3' (reverse); $\beta$-actin: 5'-CTGGAACGGTGAAGGT GACA-3' (forward) and 5'-AAGGGACTTCCTGTAACAATG CA-3' (reverse). $\beta$-actin was used as the internal control. PCR instrument on the following cycle: $94^{\circ} \mathrm{C}$ for denaturing $3 \mathrm{~min}$, $94^{\circ} \mathrm{C} 60 \mathrm{sec}, 58^{\circ} \mathrm{C} 60 \mathrm{sec}, 72^{\circ} \mathrm{C} 90 \mathrm{sec}$, a total of 35 circula- tions, $72^{\circ} \mathrm{C}$ for $10 \mathrm{~min}$, computer analysis of gene amplification, export the corresponding threshold cycle number, and the expression of $\beta$-actin as an internal control to calculate the relative gene expression levels, detecting ADAM17 mRNA expression levels of MCF-7 breast cancer cells. Each sample was detected three times with qRT-PCR, and samples obtained from three independent experiments were used to analyze the expression of relative genes.

Migration assay in vitro. Migration of cells was performed utilizing 24-well Transwell chambers with $8.0 \mu \mathrm{m}$ pore carrying polycarbonate membrane (BD Biosciences, San Jose, CA, USA) following the manufacturer's instruction. After trypsinization, cells of the ADAM17-siRNA, the nonsense siRNA, the AG1478, the LY294002, the PD0325901 and the control groups were suspended in DMEM, respectively and the cell concentration of the six groups was adjusted to $5 \times 10^{5} / \mathrm{ml}$. The concentration of AG1478, LY294002 and PD0325901 in the relevant group was $20 \mu \mathrm{M}$, respectively. Suspending cell liquid $(100 \mu \mathrm{l})$ of each group was placed in the upper compartment of the plates. Afterward, the lower compartment was filled with complete medium and chemokine mixture of $500 \mu \mathrm{l}$. After $24 \mathrm{~h}$ of incubation, the chamber polycarbonate membrane was cut, stained with eosin and mounted with neutral gum. The numbers of adherent cells in each of five random fields for a given well were counted under an optical microscope at 200 times magnification and then numbers of every five fields were numerically averaged and counted. The number of cells through the Transwell chamber was an indicator to evaluate the tending movement ability.

Growth curve. MCF-7 cells of the ADAM17-siRNA, the nonsense siRNA, the AG1478, the LY294002, the PD0325901 and the control groups were seeded at a concentration of $1.5 \times 10^{4}$ cells/well in 24 -well plates filled with the complete medium which refreshed every $24 \mathrm{~h}$. Thereafter, every day, three wells of total adherent cells from each group were trypsinized and counted utilizing a hemocytometer under the microscope, and the cell numbers of every three wells were numerically averaged. The cell growth curve was drawn after 7 days of a continuous count.

MTT assay for cell proliferation. The effects of different administrations on breast cancer cell proliferation was detected by MTT assay. In brief, MCF-7 cells during the logarithmic growth phase of the ADAM17-siRNA, the nonsense siRNA, the AG1478, the LY294002, the PD0325901 and the control group were trypsinized and seeded onto 96 -well plates at the concentration of $1 \times 10^{4} /$ well approximately, and maintained for 24,48 and $72 \mathrm{~h}$. When the culture finished, the medium was replaced by $200 \mu \mathrm{l}$ fresh medium stained with $250 \mu \mathrm{g} / \mathrm{ml}$ sterile MTT (Chemicon, Billerica, MA, USA), and then the plates were incubated at $37^{\circ} \mathrm{C}$ for further $2 \mathrm{~h}$. Afterwards, the medium was taken out carefully and $200 \mu \mathrm{l}$ DMSO was added, the reaction was sustained at $37^{\circ} \mathrm{C}$ for $15 \mathrm{~min}$. Absorbance was measured using a microplate reader (Bio-Tek ELx800; BioTek Instruments, Inc., Winooski, VT, USA) at a wavelength of $570 \mathrm{~nm}$ and subtracted from that at $450 \mathrm{~nm}$. Each experiment was conducted in triplicate and all detections were repeated in quadruplicate. 

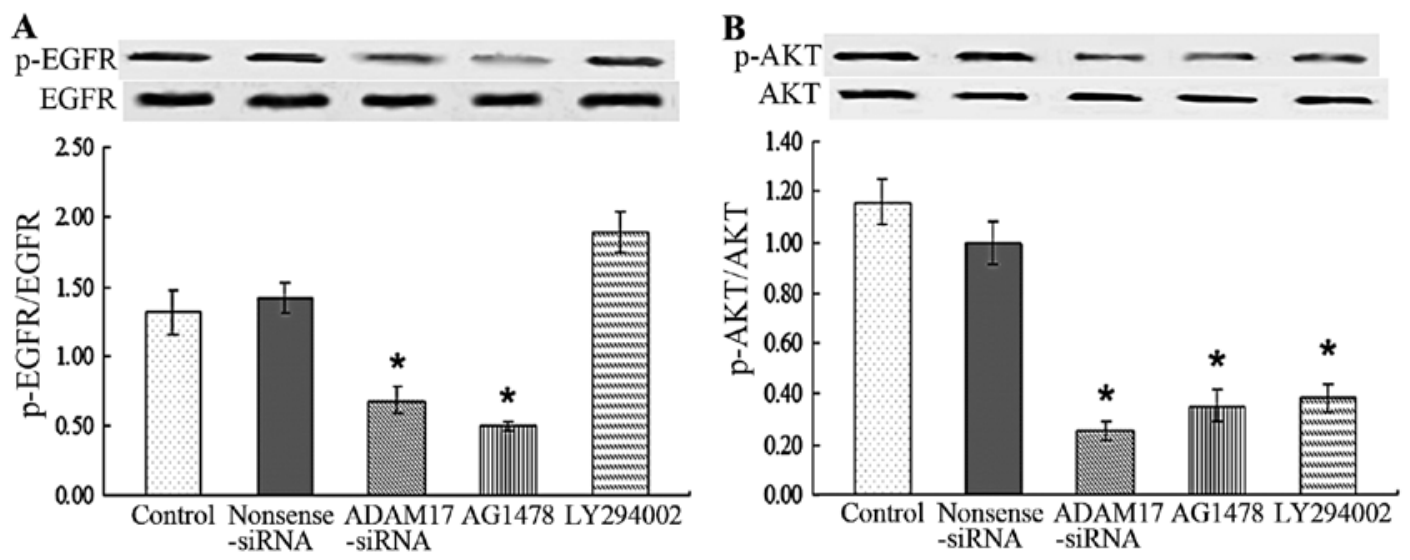

Figure 1. Representative western blot images and corresponding data illustrating p-EGFR (A) and p-AKT (B) expressions in MCF-7 cells with ADAM17siRNA, AG1478 and LY294002 administration. ${ }^{*} \mathrm{P}<0.05$ vs. control.

Treatment of ADAM17-siRNA on MCF-7 breast cancer in vivo. Female $\mathrm{Nu} / \mathrm{Nu}$ athymic mice with a body weight of 15-25 g were obtained from Beijing HFK Bioscience (Beijing, China). Estrogen $0.2 \mathrm{ml}(0.15 \mathrm{mg} / \mathrm{ml})$ was administered to the nude mice by intraperitoneal injection every day until sacrifice. MCF-7 breast cancer cells $(0.2 \mathrm{ml})\left(5 \times 10^{7} / \mathrm{ml}\right)$ were implanted subcutaneously in the right flank of the nude mice after 3 days of estrogen injection. Tumor diameter was measured with calipers and the tumor volume was calculated by the formula: (width) ${ }^{2} \mathrm{x}$ length/2. After 2 weeks of implantation, 15 nude mice which had developed tumors with an average diameter of $7 \mathrm{~mm}$ were then divided into three groups: ADAM17-siRNA group (inject ADAM17-siRNA $10 \mu \mathrm{g}$ and in vivo JetPEI ${ }^{\mathrm{TM}}$ $1.5 \mu \mathrm{l}$ ), vector group (inject equal volume of in vivo JetPEI ${ }^{\mathrm{TM}}$ ) and control group (injection equal volume of PBS). Each dose was injected at different locations around the tumor every 3 days. Mice were sacrificed on the 16th day after treatment.

Immunohistochemistry of tumors. When mice were sacrificed, the removed breast cancer transplanted tumors were fixed in $4 \%$ paraformaldehyde, paraffin-embedded, sectioned and processed for hematoxylin and eosin (H\&E) staining and immunohistochemical staining to observe under a light microscope. ADAM17 and Ki-67 were examined. A primary rabbit monoclonal antibody anti-ADAM17 (1:100; Abcam, Cambridge, MA, USA) and a primary rabbit polyclonal antibody anti-Ki-67 (1:100; Cell Signaling Technology, Danvers, MA, USA) were used, respectively, followed by HRP-conjugated secondary goat antibodies (ZSGB-Bio Co., Ltd., Beijing, China).

Results were evaluated by three pathologists in a blinded manner. The percentage of positive cells and intensity of staining in each sample should be evaluated with no less than 1000 cells and 5 high power fields. The percentage of positive cells was scored as $0(<10 \%), 1(10-30 \%), 2(31-70 \%)$ and $3(71-100 \%)$, while the staining intensity was scored as 0 (negative), 1 (weak), 2 (moderate) and 3 (strong). The whole results of immunohistochemical staining were calculated by the percentage score $\mathrm{x}$ intensity score.

Western blot analysis. Cells or tissues were washed in PBS and proteins were isolated in RIPA lysis buffer containing $2 \%$ protease inhibitor PMSF (Sigma-Aldrich). Same concentration of proteins from each group, as measured utilizing a BCA protein assay kit (Pierce, Rockford, IL, USA), were loaded onto a $10 \%$ SDS-PAGE gel after being denatured. After separation, the proteins were electro-transferred onto PVDF membranes (Invitrogen). Subsequently, the membranes were blocked with $5 \%$ non-fat milk powder in TBS-T $(10 \mathrm{mM}$, Tris-HCl, pH 7.6 and $150 \mathrm{mM} \mathrm{NaCl}, 0.1 \%$ Tween-20) at room temperature for $1 \mathrm{~h}$ and then incubated respectively with primary antibodies: anti-ADAM17 (1:1,000; Abcam), anti-EGFR (1:1,000; Cell Signaling Technology), antiphosphorylated EGFR (1:1,000; Cell Signaling Technology), anti-AKT (1:1,000; Santa Cruz Biotechnology, Santa Cruz, CA, USA) and anti-phosphorylated AKT (1:1,000; Santa Cruz Biotechnology) at $4^{\circ} \mathrm{C}$ overnight. Following washing in TBS-T, the membranes were probed with HRP-conjugated secondary antibodies (ZSGB-BIO) for $1 \mathrm{~h}$ at room temperature and immunoblots were visualized using an enhanced chemiluminescence detection kit (Amersham, Little Chalfont, UK). The experiments were conducted in triplicate.

Statistical analysis. Results are the mean \pm SD. Statistical significance was considered at $\mathrm{P}<0.05$. Comparisons among three or more groups was made by one-way ANOVA using the SPSS 13.0 software (SPSS, Inc., Chicago, IL, USA).

All experimental procedures were approved by the Institutional Animal Care and Use Committee of North China University of Science and Technology.

\section{Results}

EGFR-PI3K-AKT signaling pathway is involved in ADAM17 promoting $M C F-7$ cell migration and proliferation. To detect the mechanism by which ADAM17 promotes migration and proliferation ability of MCF-7, we evaluated the activation of EGFR. MCF-7 had high expression of phosphorylated EGFR (p-EGFR) while nonsense siRNA and LY294002 had no effect on it. Administration of ADAM17-siRNA and AG1487 significantly reduced $\mathrm{p}$-EGFR compared with control $(\mathrm{P}<0.05$; Fig. 1A). The data suggested that ADAM17 promotes migration and proliferation ability of MCF-7 cells by activating EGFR. 
A

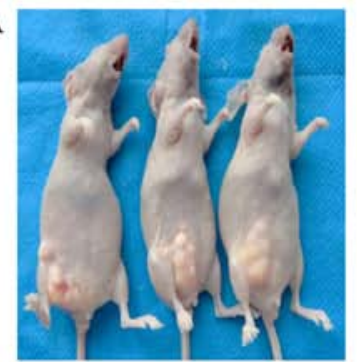

C

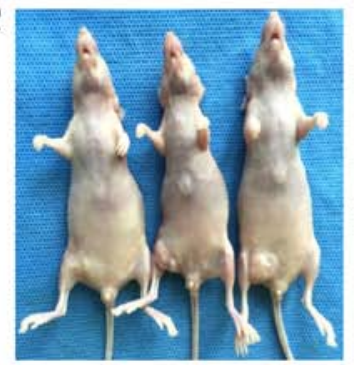

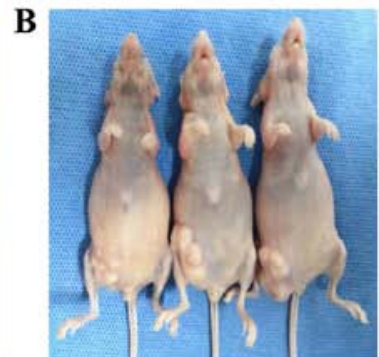

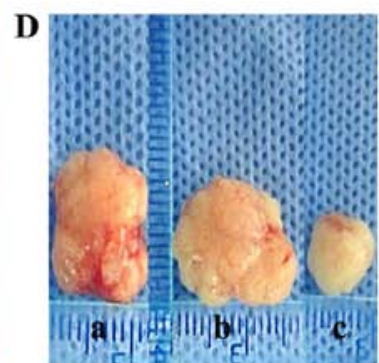

$\mathbf{E}$

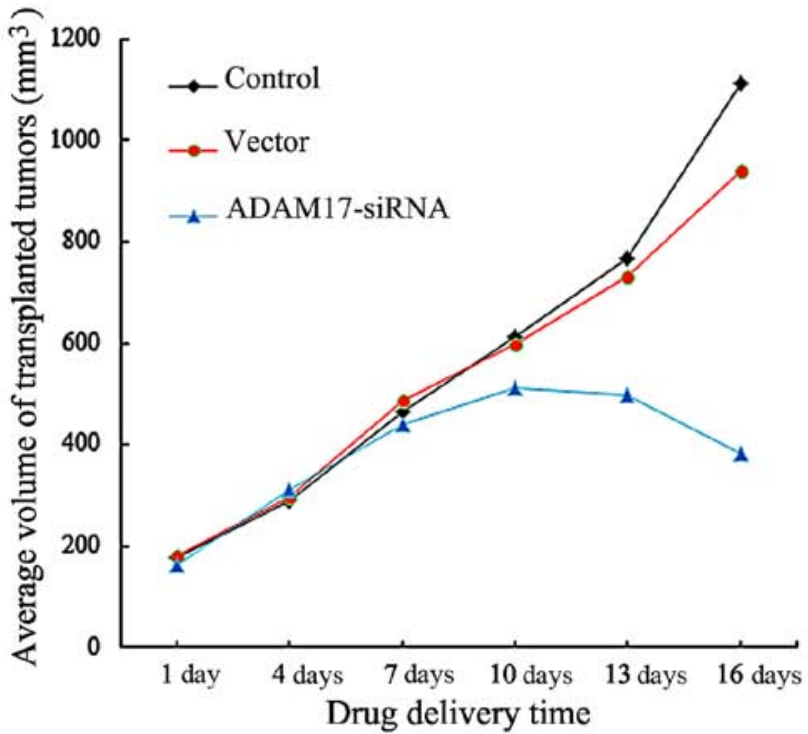

Figure 2. Tumor-bearing nude mice after sacrifice and transplanted tumor in different groups and growth curve of MCF-7 breast cancer after administration.
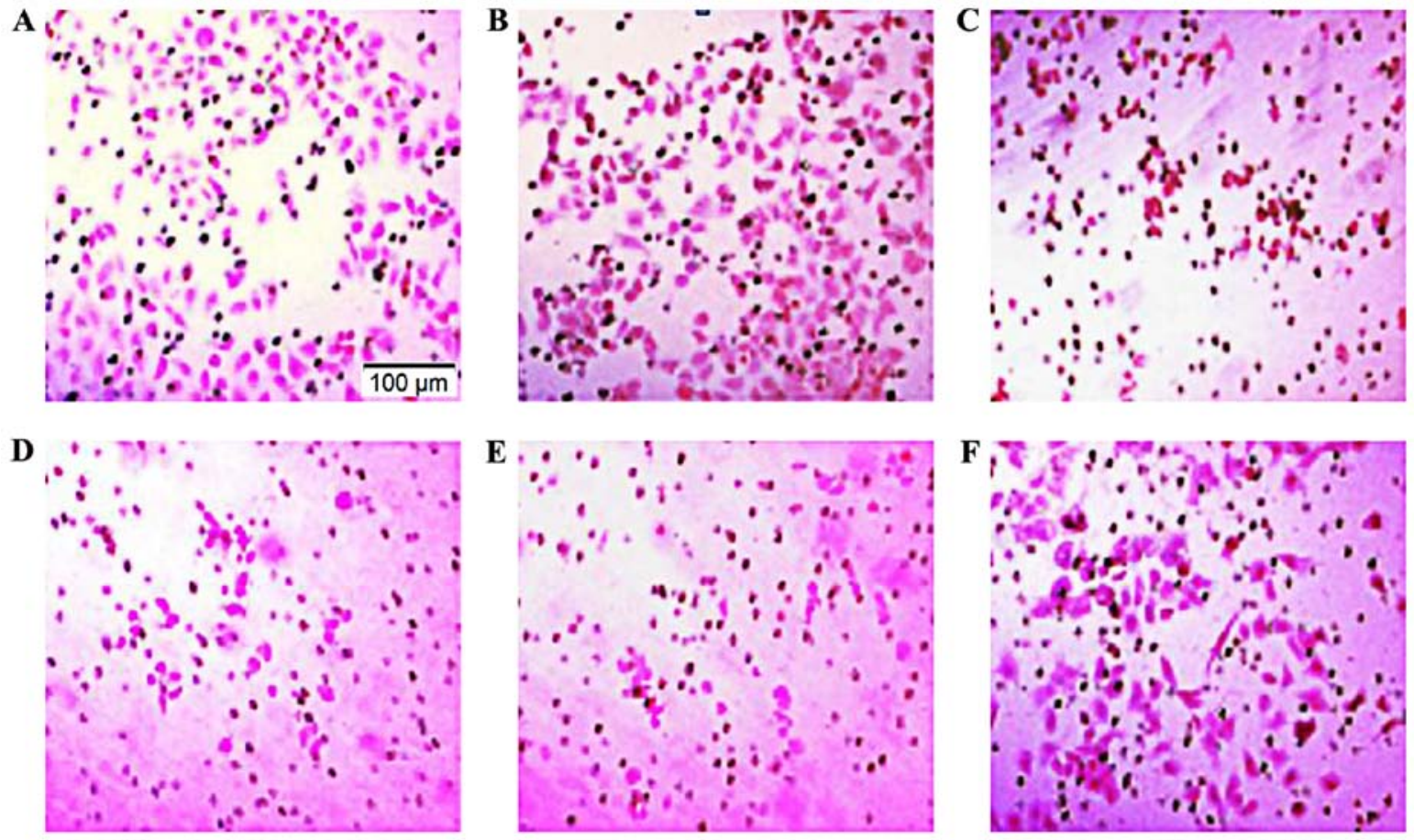

Figure 3. Migration ability of MCF-7 cells in different groups (magnification, x200) (A) Control group; (B) nonsense-siRNA group; (C) ADAM17-siRNA group; (D) AG1478 group; (E) LY294002 group; (F) PD0325901 group.

To detect whether PI3K and AKT were downstream of EGFR and mediated the promotive effect of ADAM17 on migration and proliferation of $\mathrm{MCF}-7$, we also measured the activation of AKT. The data showed that MCF-7 cells had a high level of phosphorylated AKT (p-AKT) while additional administration of ADAM17-siRNA, AG1487 and LY294002 significantly suppressed p-AKT of MCF-7 cells compared with control $(\mathrm{P}<0.05$; Fig. $1 \mathrm{~B})$. The data suggested that PI3K and AKT were activated in MCF-7 cells following the induction of EGFR, which promoted the migration and proliferation ability of MCF-7.
The tumor growth is inhibited by ADAM17-siRNA. As shown in Fig. 2, the volume of transplanted tumors in the control group (Fig. 2A) and the vector group (Fig. 2B) was significantly increased ( $a$ and $b$ in Fig. 2D); while in the ADAM17-siRNA group (Fig. 2C), tumor volume was not significantly increased (c in Fig. 2D). The tumor growth curve (Fig. 2E) was drawn according to the average volume of transplanted tumors in nude mice of different groups.

Migration of MCF-7 breast cancer cells. Migration ability of MCF-7 cells was detected by the Boyden chamber method. 


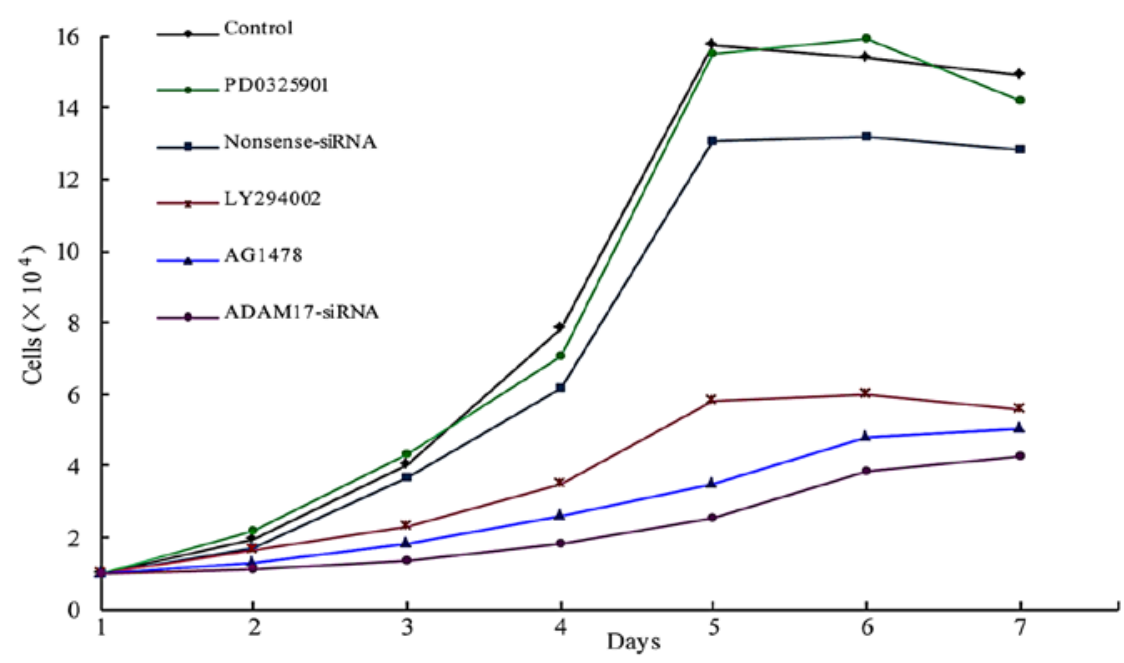

Figure 4. Growth curves of MCF-7 cells after administration.

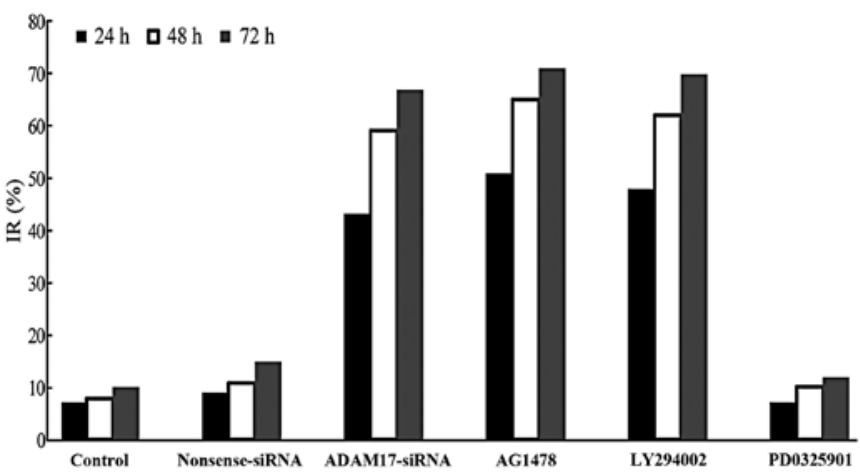

Figure 5. MCF-7 cell inhibition ratio after administration by MTT assay.

The number of migrated cells per field was $127.88 \pm 9.91$, $114.96 \pm 8.08,61.63 \pm 5.91,54.24 \pm 6.72,48.79 \pm 7.28$ and $109.82 \pm 10.42$ in the control, nonsense siRNA, ADAM17siRNA, AG1478, LY294002 and PD0325901 groups, respectively (Fig. 3). Compared with the control, there was no significant difference in the nonsense and PD0325901 groups, respectively. However, the number in the ADAM17-siRNA, AG1478 and LY294002 groups was significantly reduced $(\mathrm{P}<0.05)$. The results indicated that ADAM17 enhances MCF-7 cell migration and EGFR-PI3K-AKT signaling pathway is also involved in the migration process of MCF-7, but not EGFRMEK-ERK signaling pathway.

Growth curve. Compared with the control, there was no significant difference in the nonsense and PD0325901 groups, respectively. However, the number of the ADAM17-siRNA, AG1478 and LY294002 groups was significantly reduced ( $\mathrm{P}<0.05$; Fig. 4).

MTT assay for cell proliferation. With the extension of culture time, the proliferation ability of cells in the control group gradually enhanced. Compared with the control, there was no significant difference in the nonsense and PD0325901 groups, respectively. However, the numbers in the ADAM17siRNA, AG1478 and LY294002 groups were significantly reduced $(\mathrm{P}<0.05$; Fig. 5$)$. The results indicated that ADAM17 promotes the proliferation of MCF-7 breast cancer cells and EGFR-PI3K-AKT signaling pathway is also involved in the proliferation of MCF-7, but not EGFR-MEK-ERK signaling pathway.

ADAM17 expression in MCF-7 cells and changes induced by ADAM17-siRNA, AG1478 and LY294002 adminstration. Since ADAM17 was involved in the migration and proliferation process of MCF-7, we detected the levels of ADAM17 mRNA and protein and observed whether ADAM17 expression was affected by ADAM17-siRNA, AG1478 and LY294002 administration. As MEK had no effect on migration and proliferation of MCF-7, it was omitted in the following study. qRT-PCR analysis was used to measure mRNA levels. The $\mathrm{Ct}$ values of ADAM17 mRNA expression were corrected by $\beta$-actin and the ratio of the control group was considered as $100 \%$. Our results showed that MCF-7 cells (control) expressed ADAM17 mRNA at a high level. Compared with the control, nonsense sequence siRNA, AG1478 and LY294002 did not change ADAM17 mRNA expression, but ADAM17-siRNA significantly inhibited it $(\mathrm{P}<0.05$; Fig. 6A). The changes of ADAM17 protein expression were detected by western blot analysis. As shown in Fig. 6B, ADAM17 protein was highly expressed in MCF-7 cells. Similar to the changes of mRNA expression, ADAM17siRNA significantly decreased the level of ADAM17 protein compared with control group $(\mathrm{P}<0.05)$ although there was no difference in the nonsense sequence siRNA, AG1478 and LY294002 groups. The data suggest that ADAM17-siRNA successfully inhibits ADAM17 expression in both mRNA and protein levels, while inhibition of EGFR and PI3K has no such effect.

Pathological observation of breast cancer tissues after $H \& E$ staining. H\&E staining showed that the transplanted tumor was characterized by typical human breast invasive ductal carcinoma. Compared with the control group and the vector group, the tumor tissues in the ADAM17-siRNA group developed large areas of necrosis, where the cells were destroyed and the 
A

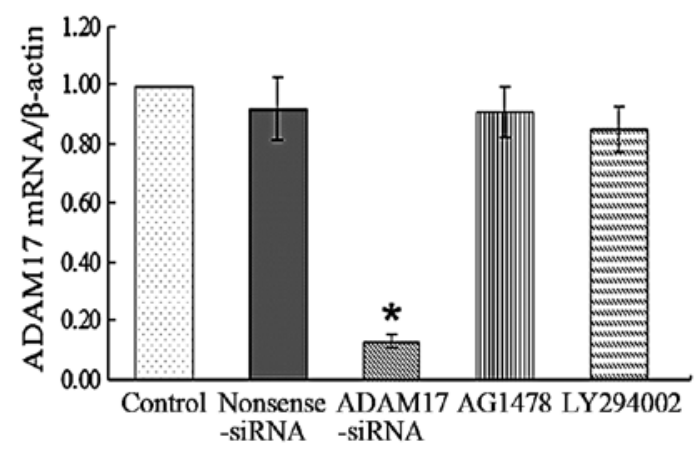

B

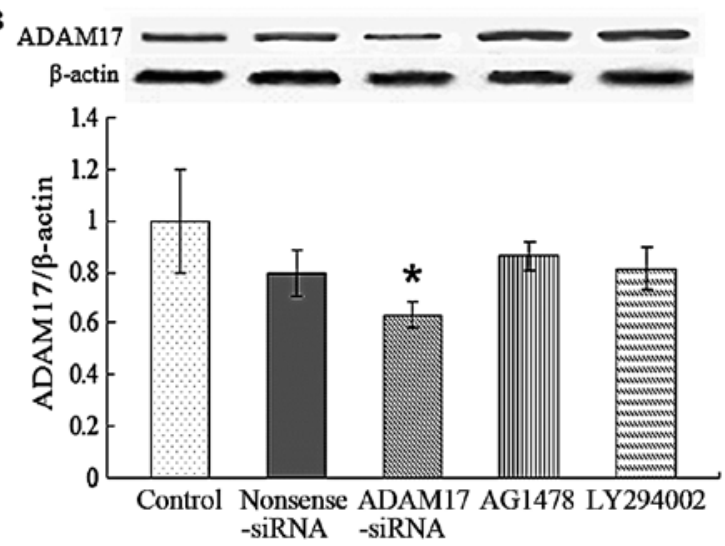

Figure 6. ADAM17 mRNA (A) and representative western blot images and corresponding data illustrating ADAM17 protein expression (B) in MCF-7 cells with ADAM17-siRNA, AG1478 and LY294002 administration. "P<0.05 vs. control.
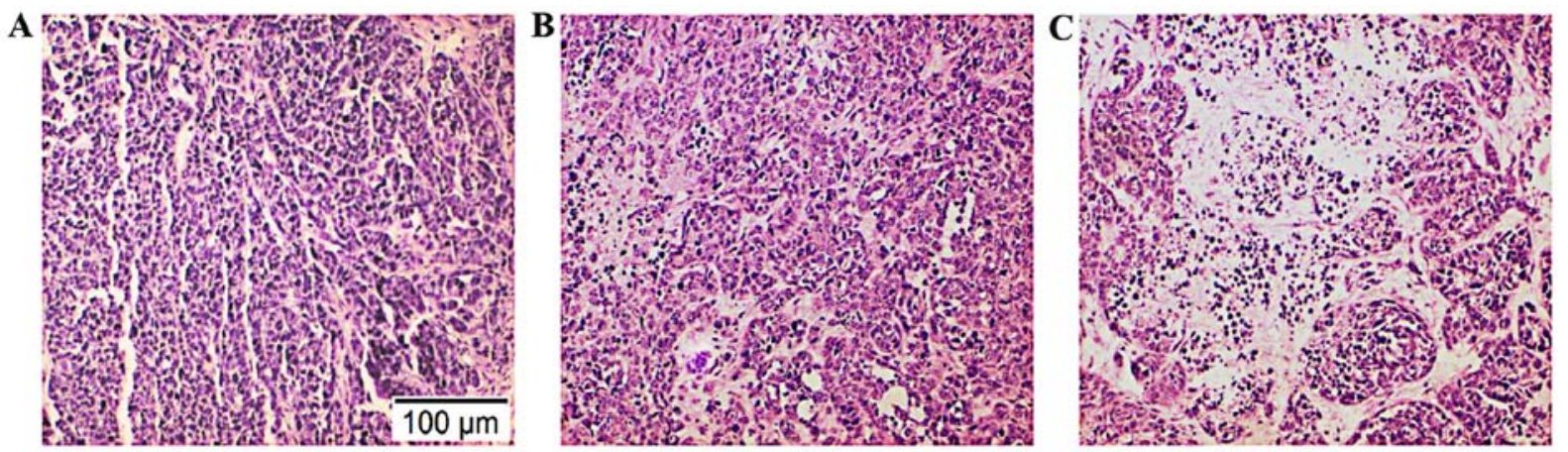

Figure 7. Representative H\&E staining images of the control group (A), the vector group (B), and the ADAM17-siRNA group (C) (magnification, x200).
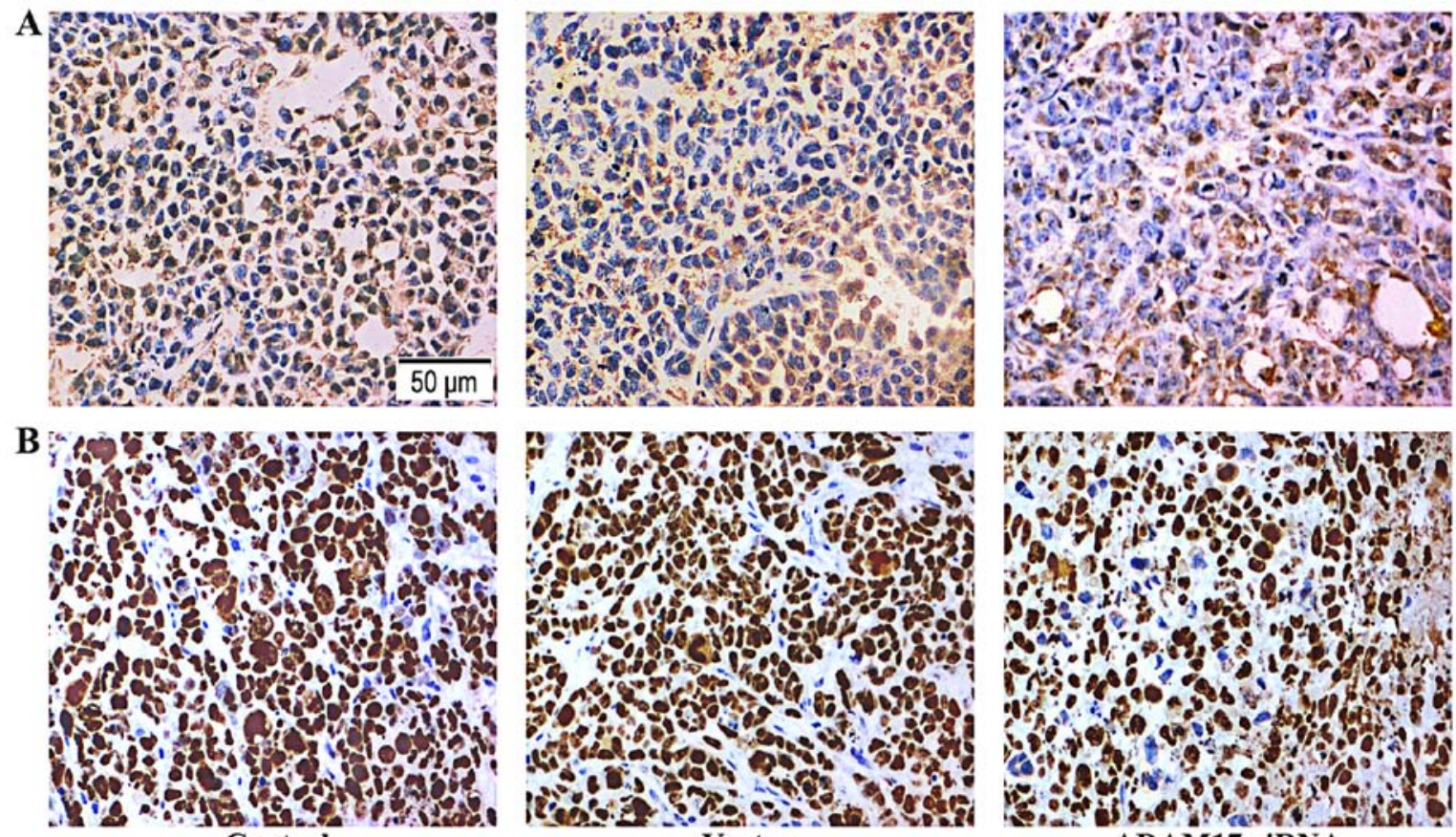

Control
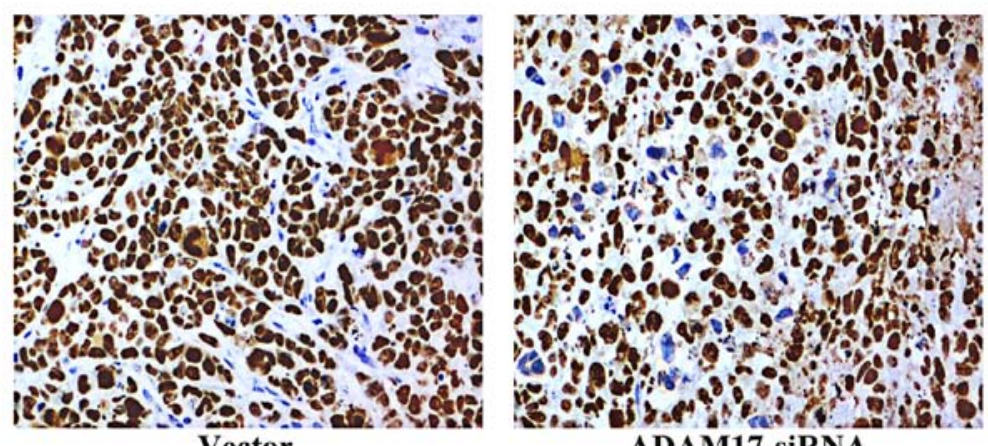

ADAM17-siRNA

Figure 8. Representative immunohistochemical images of ADAM17 (A) and Ki-67 (B) in different groups (magnification, x400).

cell structures disappeared; there was no significant difference between the vector group and the control group (Fig. 7).
Expression of ADAM17 and Ki-67 in immunohistochemistry of tumors. ADAM17 was mainly expressed in cytoplasm, 


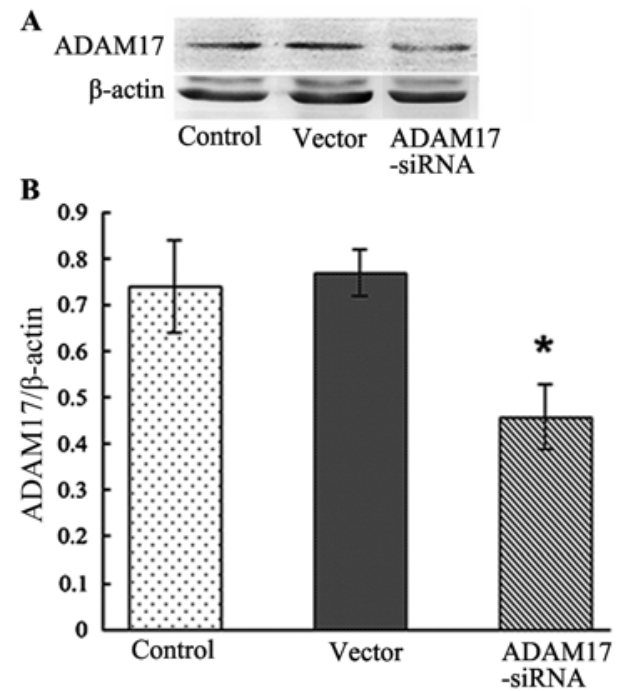

Figure 9. Representative western blot images (A) and corresponding data illustrating ADAM17 protein expression (B) in tumor tissues with ADAM17siRNA administration. ${ }^{*} \mathrm{P}<0.05$ vs. control.

as positive brown staining. The transplanted tumor staining index score in the control group was $5.57 \pm 1.8$, and in vector group was $5.29 \pm 1.89$, thus, there was no significant difference between the two groups; while the staining index of the transplanted tumor in the ADAM17-siRNA group was $1.71 \pm 1.80$, compared with the control group and the vector group, the difference had statistical significance ( $\mathrm{P}=0.001$; Fig. $8 \mathrm{~A})$. The data showed that ADAM17-siRNA inhibits the expression of ADAM17 in the transplanted tumor.

Ki-67 was mainly expressed in the nucleus, as positive brown staining. The transplanted tumor staining index score in the control group was $7.00 \pm 1.64$, and in vector group was $6.57 \pm 1.60$, thus, there was no significant difference between the two groups; while the staining index of the transplanted tumor in the ADAM17-siRNA group was 2.29+1.03, compared with the control group and the vector group, the difference had statistical significance $(\mathrm{P}<0.001$; Fig. $8 \mathrm{~B})$. The data showed that ADAM17-siRNA inhibits the expression of Ki-67 in the transplanted tumor.

ADAM17 protein expression in tumor tissues is reduced by ADAM17-siRNA. The value of ADAM17/ $\beta$-actin in the control group was $0.74 \pm 0.10$, and in the vector group was $0.77 \pm 0.05$, thus the difference between the two was not statistically significant; while the value of ADAM17/ $\beta$-actin in ADAM17siRNA group was $0.46 \pm 0.07$, compared with the control and the vector groups, the difference had statistical significance $(\mathrm{P}<0.001$; Fig. 9). This data proved that ADAM17-siRNA successfully inhibits the expression of ADAM17 protein in the transplanted tumor.

\section{Discussion}

ADAM17 is one of the most important members of the ADAM family. Besides releasing TNF- $\alpha$, ADAM17 is also conducive to the progression of disease by means of processing a number of growth factors and growth factor receptors (6). ADAM17, also known as tumor necrosis factor- $\alpha$-converting enzyme, participates in the activation of EGFR and related receptors, which is causally related to the progression of different cancers of epidermal origin (6-8).

EGFR generally has a wide range of expression in most cell types but in hematopoietic cells, moreover, its activation plays an important role in normal cell physiology processes $(20,21)$. Nonetheless, unduly increased EGFR signaling is always bound up with the growth of various malignant tumors including breast cancer (20,22-24). EGFR ligand-binding leads to receptor self-dimerization, autophosphorylation and followed activation of downstream MEK-ERK or PI3K-AKT signal pathways, which could contribute to the progression of a tumor $(12,13)$. There is plenty of research on targeting EGFR in the treatment of breast cancer (25-27). AG1478, as a specific inhibitor of EGFR tyrosine kinase, has been widely used in laboratory studies to demonstrate its antitumor function (28). Both in vitro cell models and in vivo mouse models have AG1478 prominent anti-proliferative efficacities and in some tumor cells, moreover, can heighten antitumor effects of the monoclonal antibody 806 (an anti-EGFR antibody) and cytotoxic drugs $(29,30)$. Our western blot results showed that ADAM17-siRNA and AG1478 could significantly decrease expression of $\mathrm{p}$-EGFR.

PI3K, a heterodimer made up of a p110 catalytic subunit and a p85 regulatory subunit, has been confirmed to phosphorylate the serine/threonine kinase (AKT) (31). As a downstream effector of PI3K, AKT is closely related to cell survival and anti-apoptotic signaling $(32,33)$. A recent study proved that cell signaling is mediated by PI3K-AKT through induction of ADAM17, which is involved in proliferation and migration of cancer cells (14). LY294002, which specifically inhibits the PI3K-AKT signal pathway, may compete with ATP for binding sites on PI3K, thereby suppressing AKT activation and blocking signal transduction (34). Our western blot results showed that ADAM17-siRNA, AG1478 and LY294002 significantly decreased expression of p-AKT.

Tumor cell proliferation and migration are crucial factors for the growth of malignant tumors, which are complex processes being regulated and controlled by many genes (35). Our previous results showed ADAM17-siRNA inhibited MCF-7 breast cancer cell proliferation and invasion in vitro $(18,19)$. In this project, we continued to employ MCF-7 human breast cancer cell line in investigating the effect of ADAM17 on proliferation and migration of breast cancer in vitro. Our data demonstrated that reduction of ADAM17 by siRNA significantly decreased cancer cell proliferation and migration. EGF has been demonstrated to excite the migration and proliferation of both normal and cancer cells, including normal breast epithelial cells and breast cancer cells $(36,37)$. EGFR is activated by binding ligands, such as EGF, TNF- $\alpha$, to stimulate proliferation, migration and metastasis and ADAM17 has been shown to hydrolyze these ligands (6-8). Thus, we further explored the contribution of EGFR-PI3K-AKT signal pathway or EGFRRas-Raf-MEK-ERK signal pathway to the ADAM17 induced migration and proliferation of breast cancer cells. Boyden chamber assay showed that AG1478, and LY294002 effectively blocked migration of MCF-7 cells, but PD0325901 could not affect migration of MCF-7 cells. Similarly, MTT 
assay showed that AG1478, and LY294002 also effectively reduced proliferation of MCF-7 cells, but PD0325901 could not affect proliferation of MCF-7 cells. These results indicated that ADAM17-siRNA inhibiting MCF-7 breast cancer cell migration and proliferation may be through EGFR-PI3K-AKT signal pathway, but not EGFR-Ras-RafMEK-ERK signal pathway.

We further confirmed the effect of EGFR-PI3K-AKT signal pathway on the ADAM17 induced migration and proliferation of breast cancer cells. As a competitive inhibitor of ATP binding site in the kinase domain, the quinazoline derivative AG1478 is a highly effective and specific reversible tyrosine kinase inhibitor of the EGFR $(29,30)$. We found that the activation of EGFR and its downstream AKT signal pathway could be efficiently inhibited by AG1478. Specific suppression of EGFR-PI3K-AKT signal pathway by inhibitor LY294002 also weakened the migration and proliferation ability of MCF-7 cells. The results suggested that ADAM17 contributed to cancer development via activation of EGFR-PI3K-AKT signal pathway. Kenny and Bissel (38) also reported similar findings. ADAM17 has been shown to play a critical role in the progression of EGFR dependent malignant tumors. Besides, our previous study proved ADAM17-siRNA inhibited proliferation and invasion of MCF-7 breast cancer cells in vitro $(18,19)$. These data imply that ADAM17-siRNA inhibits metastasis of breast cancer cells towards other organs.

Subsequently, we tested the antitumor effect of ADAM17-siRNA in vivo. We established transplanted tumor models of MCF-7 breast cancer cells in nude mice and used a multi-point injection of JetPEI ${ }^{\mathrm{TM}}$-ADAM17-siRNA in the tumor for treatment. The results showed that ADAM17siRNA can inhibit tumor growth and cause tissue necrosis of a tumor. Immunohistochemistry showed that the expression of ADAM17 and Ki-67 in tumor tissues was decreased. $\mathrm{Ki}-67$, a kind of alkaline protein with protease properties, as a common malignant tumor biological indicator, can be used to determine the cell proliferation activity of tumor cells, to judge the malignant degree of the tumor and to evaluate the prognosis of patients (39). Ki-67 is expressed in many kinds of tumors, especially in breast cancer tissues, and its expression level in breast cancer tissues was much higher than that in normal breast tissues (40). Our western blot results showed that ADAM17 protein expression in tumor tissues was reduced by ADAM17-siRNA. These results indicated that ADAM17-siRNA can significantly inhibit breast cancer in vivo. The way of multi-point injection in tumor is feasible in the experimental animals, but not suitable for clinical application, therefore, in a future study, we will employ bone marrow mesenchymal stem cells (BMSCs) as the carrier, to observe whether it can transport the ADAM17-siRNA to the tumor and the effect of this method on the biological behavior of breast cancer and provide a new treatment method for targeted therapy. The treatment of breast cancer targeting ADAM17 is worthy of further study.

\section{Acknowledgements}

The present study was supported by grants from the Natural Science Foundation of Hebei Province, P.R. China (no.
C2010001767) and the Tangshan Science \& Technology Bureau of Hebei Province, P.R. China (no. 14130256B).

\section{References}

1. Krieger N, Bassett MT and Gomez SL: Breast and cervical cancer in 187 countries between 1980 and 2010. Lancet 379: 1391-1392, 2012.

2. Caiazza F, McGowan PM, Mullooly M, Murray A, Synnott N, O'Donovan N, Flanagan L, Tape CJ, Murphy G, Crown J, et al: Targeting ADAM-17 with an inhibitory monoclonal antibody has antitumour effects in triple-negative breast cancer cells. Br J Cancer 112: 1895-1903, 2015.

3. Ocaña A, Amir E, Seruga B, Martin M and Pandiella A: The evolving landscape of protein kinases in breast cancer: Clinical implications. Cancer Treat Rev 39: 68-76, 2013.

4. Zhang P, Shen M, Fernandez-Patron C and Kassiri Z: ADAMs family and relatives in cardiovascular physiology and pathology. J Mol Cell Cardiol 93: 186-199, 2015.

5. Bolger JC and Young LS: ADAM22 as a prognostic and therapeutic drug target in the treatment of endocrine-resistant breast cancer. Vitam Horm 93: 307-321, 2013.

6. Rose-John S: ADAM17, shedding, TACE as therapeutic targets. Pharmacol Res 71: 19-22, 2013.

7. Lu Y, Jiang F, Zheng X, Katakowski M, Buller B, To SS and Chopp M: TGF- $\beta 1$ promotes motility and invasiveness of glioma cells through activation of ADAM17. Oncol Rep 25: 1329-1335, 2011.

8. Santiago-Josefat B, Esselens C, Bech-Serra JJ and Arribas J: Post-transcriptional up-regulation of ADAM17 upon epidermal growth factor receptor activation and in breast tumors. J Biol Chem 282: 8325-8331, 2007.

9. Murphy G: The ADAMs: Signalling scissors in the tumour microenvironment. Nat Rev Cancer 8: 929-941, 2008.

10. Rego SL, Helms RS and Dréau D: Tumor necrosis factor-alphaconverting enzyme activities and tumor-associated macrophages in breast cancer. Immunol Res 58: 87-100, 2014.

11. Maretzky T, Zhou W, Huang XY and Blobel CP: A transforming Src mutant increases the bioavailability of EGFR ligands via stimulation of the cell-surface metalloproteinase ADAM17. Oncogene 30: 611-618, 2011.

12. Zheng X, Jiang F, Katakowski M, Lu Y and Chopp M: ADAM17 promotes glioma cell malignant phenotype. Mol Carcinog 51: $150-164,2012$

13. Xiao LJ, Lin P, Lin F, Liu X, Qin W, Zou HF, Guo L, Liu W, Wang SJ and Yu XG: ADAM17 targets MMP-2 and MMP-9 via EGFR-MEK-ERK pathway activation to promote prostate cancer cell invasion. Int J Oncol 40: 1714-1724, 2012.

14. Zheng X, Jiang F, Katakowski M, Zhang ZG, Lu QE and Chopp M: ADAM17 promotes breast cancer cell malignant phenotype through EGFR-PI3K-AKT activation. Cancer Biol Ther 8: 1045-1054, 2009.

15. McGowan PM, McKiernan E, Bolster F, Ryan BM, Hill AD, McDermott EW, Evoy D, O'Higgins N, Crown J and Duffy MJ: ADAM-17 predicts adverse outcome in patients with breast cancer. Ann Oncol 19: 1075-1081, 2008.

16. Han X, Yang XF, Zhang XP, Sun Y, Zhao JH and Zhao GM: Expression of ADAM17 in tissue of breast cancer and its clinical significance. Modern Journal of Integrated Traditional Chinese and Western Medicine 19: 2486-2488, 2010.

17. Yang XF, Zhang XP, Zhao GM, Sun Y and Han X: Clinical significance of ADAM-17 protein expression in invasive breast cancer. Shandong Med J 51: 84-85, 2011.

18. Yang WJ, Zhang XP, Jiao JM, Zhao GM, Lu YQ and Hu BS: Inhibitory effects of ADAM17-siRNA on the invasion of human MCF-7 breast cancer. Tianjin Med J 39: 1045-1407, 2011.

19. Lu YQ, Zhao GM and Zhang XP: Effects of ADAM17-siRNA on the proliferation of human MCF-7 breast cancer. Modern J Integrated Traditional Chinese and Western Med 20: 3003-3005, 2011.

20. Brand TM, Iida M, Luthar N, Starr MM, Huppert EJ and Wheeler DL: Nuclear EGFR as a molecular target in cancer. Radiother Oncol 108: 370-377, 2013.

21. Dienstmann R, Braña I, Rodon J and Tabernero J: Toxicity as a biomarker of efficacy of molecular targeted therapies: Focus on EGFR and VEGF inhibiting anticancer drugs. Oncologist 16: 1729-1740, 2011. 
22. Azuaje F, Tiemann K and Niclou SP: Therapeutic control and resistance of the EGFR-driven signaling network in glioblastoma. Cell Commun Signal 13: 23, 2015.

23. Lee CC, Shiao HY, Wang WC and Hsieh HP: Small-molecule EGFR tyrosine kinase inhibitors for the treatment of cancer. Expert Opin Investig Drugs 23: 1333-1348, 2014.

24. Lorusso V, Forcignano R, Cinieri S, Tinelli A, Porcelli L, Quatrale AE and Chiuri VE: Which role for EGFR therapy in breast cancer? Front Biosci (Schol Ed) 4: 31-42, 2012.

25. Lluch A, Eroles P and Perez-Fidalgo JA: Emerging EGFR antagonists for breast cancer. Expert Opin Emerg Drugs 19: 165-181, 2014

26. Howe LR and Brown PH: Targeting the HER/EGFR/ErbB family to prevent breast cancer. Cancer Prev Res (Phila) 4: 1149-1157, 2011.

27. Davis NM, Sokolosky M, Stadelman K, Abrams SL, Libra M, Candido S, Nicoletti F, Polesel J, Maestro R, D'Assoro A, et al: Deregulation of the EGFR/PI3K/PTEN/Akt/mTORC1 pathway in breast cancer: Possibilities for therapeutic intervention. Oncotarget 5: 4603-4650, 2014.

28. Li P, Torossian A, Zhang Q, Xu WC and Fu S: Inhibition of phosphoinositide 3-kinase enhances the cytotoxicity of AG1478, an epidermal growth factor receptor inhibitor, in breast cancer cells Med Oncol 29: 3258-3264, 2012.

29. Caja L, Sancho P, Bertran E, Ortiz C, Campbell JS, Fausto N and Fabregat I: The tyrphostin AG1478 inhibits proliferation and induces death of liver tumor cells through EGF receptordependent and independent mechanisms. Biochem Pharmacol 82: 1583-1592, 2011.

30. Zhang YG, Du Q, Fang WG, Jin ML and Tian XX: Tyrphostin AG1478 suppresses proliferation and invasion of human breast cancer cells. Int J Oncol 33: 595-602, 2008.

31. Ke XY, Wang Y, Xie ZQ, Liu ZQ, Zhang CF, Zhao Q and Yang DL: LY294002 enhances inhibitory effect of gemcitabine on proliferation of human pancreatic carcinoma PANC-1 cells. J Huazhong Univ Sci Technolog Med Sci 33: 57-62, 2013.

32. Tian S, Chang W, Du H, Bai J, Sun Z, Zhang Q, Wang H, Zhu G, Tao K and Long Y: The interplay between GRP78 expression and Akt activation in human colon cancer cells under celecoxib treatment. Anticancer Drugs 26: 964-973, 2015.
33. Zhang Y, Zheng L, Ding Y, Li Q, Wang R, Liu T, Sun Q, Yang H, Peng S, Wang W, et al: MiR-20a induces cell radioresistance by activating the PTEN/PI3K/Akt signaling pathway in hepatocellular carcinoma. Int J Radiat Oncol Biol Phys 92: 1132-1140, 2015.

34. Chen P, Wu J, Yuan Q, Jiang X and Huang H: The synergistic killing of AML cells co-cultured with HS-5 bone marrow stromal cells by As2O3 and the PI3K/Akt signaling pathway inhibitor LY294002. Pharmazie 70: 322-327, 2015.

35. Wang Y and Lazo JS: Metastasis-associated phosphatase PRL-2 regulates tumor cell migration and invasion. Oncogene 31: 818-827, 2012.

36. Whitsett TG, Cheng E, Inge L, Asrani K, Jameson NM, Hostetter G, Weiss GJ, Kingsley CB, Loftus JC, Bremner R, et al: Elevated expression of Fn14 in non-small cell lung cancer correlates with activated EGFR and promotes tumor cell migration and invasion. Am J Pathol 181: 111-120, 2012.

37. Han J, Xie Y, Lan F, Yu Y, Liu W, Chen J, Zheng F, Ouyang X, Lin X, Lin Y, et al: Additive effects of EGF and IL-1 $\beta$ regulate tumor cell migration and invasion in gastric adenocarcinoma via activation of ERK1/2. Int J Oncol 45: 291-301, 2014.

38. Kenny PA and Bissell MJ: Targeting TACE-dependent EGFR ligand shedding in breast cancer. J Clin Invest 117: 337-345, 2007.

39. Tanei T, Shimomura A, Shimazu K, Nakayama T, Kim SJ, Iwamoto T, Tamaki Y and Noguchi S: Prognostic significance of Ki67 index after neoadjuvant chemotherapy in breast cancer. Eur J Surg Oncol 37: 155-161, 2011.

40. Yoshioka T, Hosoda M, Yamamoto M, Taguchi K, Hatanaka KC, Takakuwa E, Hatanaka Y, Matsuno Y and Yamashita H: Prognostic significance of pathologic complete response and Ki67 expression after neoadjuvant chemotherapy in breast cancer. Breast Cancer 22: 185-191, 2015. 